\title{
Sepsistherapie 2007 - zurück auf dem Boden der Tatsachen
}

\author{
Tobias Welte
}

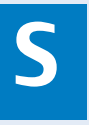

chwere Infektionskrankheiten sind zu einem Großteil die Erkrankungen des alten Menschen. Die sich schnell verändernde Demografie und die Intensivierung der Therapie bei früher schnell zum Tode führenden Erkrankungen hat weltweit zu einem dramatischen Anstieg der Sepsisprävalenz beigetragen. Ein Ende dieser Entwicklung ist nicht absehbar. Schwere Sepsis (also Sepsis mit Organversagen) und septischer Schock (also Sepsis mit katecholaminbedürftigem Kreislaufversagen) sind dabei immer noch mit einer Sterblichkeit von mehr als $50 \%$ belastet und stellen hierdurch eines der wesentlichen medizinischen und ökonomischen Probleme der Intensivmedizin dar.

Über Jahrzehnte waren alle Versuche gescheitert, mittels molekularer, in die Pathophysiologie der Sepsis eingreifender Therapiekonzepte - als Beispiel seien die Endotoxinantikörper oder Antikörper gegen TNF- $\alpha$ genannt - eine Verbesserung der Prognose von Sepsispatienten zu erreichen. 2001 sah es dann so aus, als sei auf breiter Front eine Verbesserung für Sepsispatienten erreicht worden. Aktiviertes Protein C (Drotrecogin Alpha, Xigris $\left.{ }^{\circledR}\right)$ bewirkte als erstes Medikament in einer randomisierten, kontrollierten multizentrischen Studie, dem so genannten PROWESS Trial, eine deutlich besseres Überleben als Plazebo (absolute Reduktion der Sterblichkeit 6,1\%, relative 19,4\%).

Weniger später fand Annane, dass septische Patienten häufig unter einer relativen Nebennierenrindeninsuffizienz leiden. Eine Substitution von Hydrocortison und Mineralocorticoiden bei Patienten im septischen Schock verbesserte die hämodynamische Situation und verringerte ihren Katecholaminbedarf. Dies ging mit einer absoluten Reduktion der Sterblichkeit um $10 \%$ einher.

Die Arbeitsgruppe um G. van den Berghe aus Leuven konnte in jahrelanger Forschungsarbeit belegen, dass erhöhte Blutzuckerwerte unabhängig davon, ob ein bekannter Diabetes mellitus vorliegt, bei Intensivpa- tienten zu einer Verschlechterung der Grundkrankheit führen. Mit einer strengen Kontrolle des Blutzuckerspiegels durch eine intensivierte Insulintherapie (angestrebter Blutzuckerwert $80-110 \mathrm{mg} \%$ ) gelang es der Gruppe, bei chirurgischen Intensivpatienten einen Rückgang infektiöser Erkrankungen und vor allem auch septischer Infektionen zu erzielen und damit die Prognose der Patienten deutlich zu bessern.

Bereits im Jahre 2000 hat das ARDS-Network zeigen können, dass eine Reduktion des Atemzugvolumens auf 4-6 ml/kg Körpergewicht bei Patienten mit ARDS, eine der Hauptkomplikationen einer Sepsis, zu einer Reduktion der Sterblichkeit um 8,8\% absolut führte.

Nach Jahren der Stagnation im Bereich der Antibiotikaentwicklung wurde 2003 mit dem Oxazolidinon Linezolid eine vollkommen neue Substanzgruppe in den Markt eingeführt, die bei Patienten mit durch Methicillin-resistentem S. aureus (MRSA) - einem weltweit zunehmend wichtigen Problemkeim - ausgelöster Pneumonie deutlich bessere klinische Ergebnisse zeigte. Auch wenn es keine diesbezüglichen Studien zur Sepsis gab, erhöhte sich die Hoffnung auf eine zuverlässigere kausale Therapie, zumal Pneumonien als wichtigste Infektionsquelle für Septitiden anzusehen sind. Weitere gegen MRSA wirksame Antibiotika folgten 2006 mit Tigecyclin und Daptomycin. Letzteres bekam inzwischen sogar eine Zulassung zur Behandlung der Staphylokokkenbakteriämie unklaren Ursprungs.

Angetrieben durch den Erfolg dieser Studien wurde die internationale Surviving Sepsis Campaign gegründet, die das ehrgeizige Ziel ausgab, die Sepsissterblichkeit durch gemeinsame wissenschaftliche und klinische Anstrengungen in absehbarer Zeit um 25\% zu senken. Aktuelles Wissen sollte durch Veröffentlichung einer Leitlinie weltweit in die Klinik implementiert und vereinheitlicht werden. In Deutschland gründete sich unter Leitung von Konrad Reinhart aus Jena die Deutsche Sepsis Gesellschaft. Das Bundesministerium für Bil- 
dung und Forschung (BMBF) unterstützte mit der Gründung des Kompetenznetzes Sepsis (SEPNET) die weitere Sepsisforschung in Deutschland und rückte das Krankheitsbild in nie dagewesener Weise in das Licht der Öffentlichkeit. Sechs Jahre später ist es an der Zeit, Bilanz zu ziehen. Und diese fällt nüchterner aus als erwartet.

Aktiviertes Protein $C$ hat sich in einer von der FDA geforderten Studie bei leichter erkrankten Patienten nicht bewährt. Es gibt weiterhin eine Indikation bei schwer kranken Sepsispatienten mit Zwei- oder MehrOrganversagen und einem APACHE Score $\geq 24$. Aufgrund der Nebenwirkungen durch die fibrinolytische Aktivität der Substanz kommt aktiviertes Protein C jedoch nur noch bei ausgewählten Patienten infrage. Die allgemeine Verunsicherung bei den Behandlern hat dazu geführt, dass aktiviertes Protein C, auch wenn es indiziert ist, nur selten eingesetzt wird.

Die positiven Ergebnisse der Annane-Studie führten zu einem generellen Einsatz von Hydrocortison. Die demnächst wahrscheinlich im New England Journal of Medicine erscheinende CORTICUS-Studie belegt jedoch, dass, wenn überhaupt, nur Patienten im septischen Schock profitieren. Ein zu breiter Einsatz bei Patienten ohne schwere hämodynamische Problematik erhöht eher nur die Nebenwirkungen dieser Therapie.

Die ebenfalls demnächst im New England Journal erscheinende VISEP Studie von SEPNET konnte die (allerdings nicht bei Sepsispatienten erhobenen) Vorteile der intensivierten Insulintherapie nicht bestätigen. Im Gegenteil zeigte sich eine erhöhte Rate an Hypoglykämien. In den deutschen Empfehlungen zur Sepsistherapie wird daher eine moderate Blutzuckereinstellung (120-150 mg\%) empfohlen, ohne dass deren Nutzen belegt wäre.

Die Ergebnisse des ARDS-Networks zur Beatmung mit niedrigen Atemzugvolumina wurden inzwischen in weiteren Untersuchungen bestätigt. Weitere Studien zur Frage der PEEP-Einstellung, des Recruitment-Ma- növers oder einer Corticoidtherapie zur Verhinderung eines chronischen Lungenschadens blieben negativ. Obwohl das Prinzip des „low-tidal-volumes“ praktisch jedem Intensivmediziner bekannt ist, konnte die Deutsche Sepsis Gesellschaft in einer in Kürze wahrscheinlich in Critical Care Medicine erscheinenden Arbeit zeigen, dass im Alltag nur weniger als $10 \%$ der deutschen Intensivstationen nach diesem Prinzip verfahren. Leitlinien führen eben nicht automatisch zu einer Verbesserung im praktischen Alltag.

Auch die Euphorie mit den neuen Antibiotika ist weitgehend verflogen. Daptomycin und Tigecyclin waren bei Pneumonie den klassischen Antibiotika unterlegen, Linezolid offenbarte eine Reihe schwerwiegender Nebenwirkungen wie Anämie, Thrombozytopenie und Polyneuropathie und musste anwendungsbeschränkt werden.

Was bleibt also von der Euphorie des Jahres 2001? Ich glaube eine ganze Menge. Die Studien der damaligen Jahre wurden überwertet. Fälschlicherweise wurden Ergebnisse aus anderen Indikationsbereichen auf die Sepsis übertragen oder Ergebnisse von Patienten mit septischem Schock generalisiert. Die letzten Jahre haben diese Missstände weitgehend korrigiert. Positiv ist jedoch zu vermerken, dass Sepsis weltweit als Krankheitsbild intensiver wahrgenommen wird als je zuvor. Dies führt zu intensiveren Forschungsaktivitäten, aber auch durch bessere Fort- und Weiterbildung zu einer besseren klinischen Versorgung. Weltweit entstanden Netzwerke, die die Durchführung sinnvoller, hochqualitativer Studien mit neuen Therapieoptionen (wie z.B. Selen) erlauben. Dass wir in Deutschland mit SEPNET eines dieser international hochbewerteten Netze aufbauen konnten, darf als großer Erfolg - persönlich für Konrad Reinhart, generell jedoch für die gesamte deutsche Intensivmedizin - gefeiert werden.

$25 \%$ Rückgang der Sterblichkeit wird nicht - wie anfangs geträumt - in wenigen Jahren zu realisieren sein. Die Struktur, die Fortschritt ermöglicht, hat sich jedoch erheblich verbessert. 
